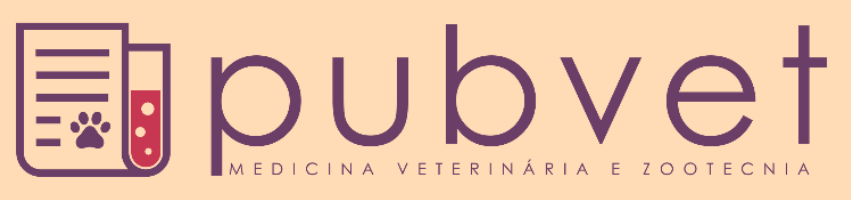

https://doi.org/10.31533/pubvet.v16n02a1042.1-5

\title{
Ovariectomia por videolaparoscopia por meio de dois portais: Relato de caso
}

\author{
Roberta Soares Braga ${ }^{1 \oplus}$, Matheus Collantes Bahia ${ }^{2 \oplus}$, Marcelo Alves Herdy ${ }^{3 \oplus}$ \\ ${ }^{1}$ Discente de Medicina Veterinária da Universidade do Grande Rio - Campus Duque de Caxias. Rio de Janeiro, RJ Brasil - \\ ${ }^{2}$ Médico veterinário autônomo e docente da empresa DOK hospital veterinário - Rio de Janeiro, RJ Brasil \\ ${ }^{3}$ Docente do curso de Medicina Veterinária da Universidade do Grande Rio - Campus Duque de Caxias, RJ Brasil \\ *Autor para correspondência. E-mail: roberta_sbraga@hotmail.com
}

Resumo. O presente estudo teve como objetivo descrever as vantagens da videolaparotomia para o paciente e como ela é factível na medicina veterinária. Onde foi relatada uma ovariectomia (remoção cirúrgica dos ovários) eletiva por vídeo cirurgia após exames préoperatórios sem alterações. A cirurgia por vídeo é realizada por meio de microcâmeras e é classificada como um procedimento cirúrgico minimamente invasivo, não ultrapassando $1 \mathrm{~cm}$ de incisão na maioria dos casos. A videocirurgia em cães e gatos possibilita diagnóstico e tratamento de diversas afecções, e sua utilização apresenta potencial crescimento na Medicina Veterinária. Pode-se concluir que a vídeo cirurgia é um procedimento significativo quando se trata de trans e pós-operatório e que cada vez mais cresce na medicina veterinária.

Palavras-chave: Cão, castração, ovariectomia, videocirurgia

\section{Video laparoscopic ovariectomy two-portal access: Case report}

Abstract. The present study aims to describe the advantages of videolaparotomy for the patient and how it is feasible in veterinary medicine. Where an elective ovariectomy by video surgery was reported after preoperative exams without changes. Video surgery is performed using micro cameras and is classified as a minimally invasive surgical procedure, not exceeding $1 \mathrm{~cm}$ incision in most cases. Video surgery in dogs and cats allows diagnosis and treatment of several conditions, and its use has potential growth in Veterinary Medicine. It can be concluded that video surgery is a significant procedure when it comes to trans and postoperative and that it is increasingly growing in veterinary medicine.

Keywords: Dog, castration, ovariectomy, video surgery

\section{Ovariectomía videolaparoscopia a través de dos portales: Reporte de caso}

Resumen. El presente estudio tuvo como objetivo describir las ventajas de la video laparotomía para el paciente y cómo es factible en medicina veterinaria. Donde se informó una ovariectomía electiva (extirpación quirúrgica de los ovarios) mediante video cirugía después de exámenes preoperatorios sin cambios. La video cirugía se realiza mediante microcámaras y se clasifica como un procedimiento quirúrgico mínimamente invasivo, no superando $1 \mathrm{~cm}$ incisión en la mayoría de los casos. La video cirugía en perros y gatos permite el diagnóstico y tratamiento de diversas afecciones, y su uso tiene potencial de crecimiento en Medicina Veterinaria. Se puede concluir que la video cirugía es un procedimiento significativo a la hora de trans y postoperatorio y que está creciendo cada vez más en medicina veterinaria.

Palabras clave: Perro, castración, ovariectomía, video cirugía 


\section{Introdução}

O acesso abdominal através de reduzidas incisões, conhecido por minilaparotomia, introduziu o conceito de cirurgia minimamente invasiva. $\mathrm{O}$ estudo dos procedimentos minimamente invasivos teve início em 1806 com a criação da primeira peça para fins de visualização das cavidades corporais, o instrumento chamado de "Lichtleiter" que tinha como fonte luminosa uma vela de cera e foi inventada por Phillips Bozzini em 1806, que a experimentou em animais. O primeiro exame endoscópico com insuflação da cavidade peritoneal (pneumoperitôneo) foi realizado experimentalmente em cães, pelo alemão George Kelling em 1901 com um cistocópio, surgindo nesta ocasião o termo "celioscopia" (Ferraz \& Lacombe, $\underline{2003}$ ). Em meados da década de 1980, a evolução tecnológica propiciou o desenvolvimento de equipamentos que determinaram expansão das possibilidades da técnica que passou de diagnóstica a terapêutica, e a grande explosão da videocirurgia ocorreu através do desenvolvimento da microcâmera e seu acoplamento a óptica laparoscópica. Já em 1985 foi descrita a primeira esterilização laparoscópica em cães (Nacul et al., 2015; Venzin, 2014).

Segundo Carvalho et al. (2007) a esterilização cirúrgica eletiva de cadelas e gatas é um dos procedimentos mais comumente realizados na prática veterinária devido aos seus potenciais benefícios como controle populacional, prevenção de doenças do trato reprodutivo, atenuação de comportamentos indesejáveis associados à atividade hormonal e redução populacional de cães e gatos.

A ovariectomia laparoscópica é um método cirúrgico simples e bem estabelecido, por isso, esta técnica cirúrgica é a melhor forma de expandir a curva de aprendizado e se familiarizar com o equipamento, sendo um procedimento cirúrgico de rotina para castrar na prática de pequenos animais (Shariati et al., 2014; Venzin, 2014).

Desde o início da década de 1990, a cirurgia minimamente invasiva, especificamente a laparoscopia, tem sido amplamente adotada como uma alternativa à laparotomia em cirurgia humana, e se tornou um padrão de cuidado para o tratamento de muitas doenças. Numerosos estudos demonstraram que os procedimentos laparoscópicos resultam em estadias hospitalares mais curtas e melhores resultados cirúrgicos quando comparados com a cirurgia abdominal aberta (Manassero \& Viateau, 2018).

A vídeo cirurgia é um recurso para cirurgias minimamente invasivas que possibilita diminuir o trauma cirúrgico, risco de infecção, dor pós operatória, tempo de recuperação, e ao longo dos anos vem sendo usada na Medicina Veterinária (

O objetivo desse estudo foi relatar um caso de ovariectomia em uma cadela por videolaparoscopia por meio de dois portais.

\section{Relato de caso}

Uma cadela, SRD com dois anos de idade pesando sete $\mathrm{kg}$ foi encaminhada ao hospital veterinário, para realizar uma ovariectomia eletiva após exame físico, eletrocardiograma, raio-x de tórax e exames laboratoriais (hemograma e bioquímica) sem alterações. No preparo do paciente realizou-se ampla tricotomia da região abdominal, e a antissepsia foi feita com o uso do clorexidina degermante $2 \%$ seguido do clorexidina alcoólico $2 \%$.

No protocolo anestésico utilizado foi administrado opióide metadona $0,25 \mathrm{mg} / \mathrm{kg}$ e a indução anestésica foi realizada com propofol $1 \mathrm{mg} / \mathrm{kg}$, cetamina $1 \mathrm{mg} / \mathrm{kg}$ e midazolan $0,5 \mathrm{mg} / \mathrm{kg}$, e a manutenção foi realizada com isoflurano.

$\mathrm{O}$ paciente foi posicionado em decúbito dorsal, sendo realizados dois acessos pela técnica aberta. $\mathrm{O}$ primeiro acesso se deu através de mini celiotomia $2 \mathrm{~cm}$ caudalmente à cicatriz umbilical para a inserção do portal de $5 \mathrm{~mm}$, por onde a ótica de $5 \mathrm{~mm}$ foi passada e a mangueira de insuflação colocada (Figura 1). Foi estabelecido pneumoperitôneo com pressão intracavitária limitada A12 mmHG pelo insuflador Olympus UHI-3 ${ }^{\circledR}$ (Figura 2).

Após a inspeção da cavidade foi realizada a segunda incisão de 5 a $6 \mathrm{~cm}$ caudal ao primeiro acesso, sendo feita de maneira vídeo assistida uma perfuração direta guiada pela ótica (Stryker endoscopy 6000 Light source ${ }^{\circledR}$ - Figura 3) e digital câmera (Stryker $988^{\circledR}$ - Figura 4), para a captura de vídeo digital. Inserindo assim o segundo portal de $5 \mathrm{~mm}$. 


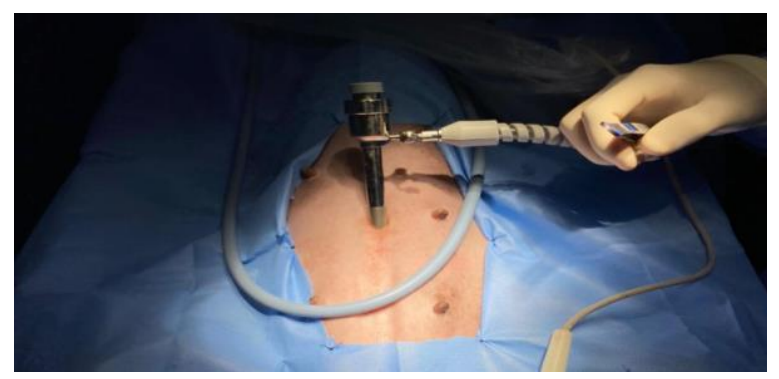

Figura 1. Primeiro acesso com a mangueira de insuflação acoplada ao portal de $5 \mathrm{~mm}$.

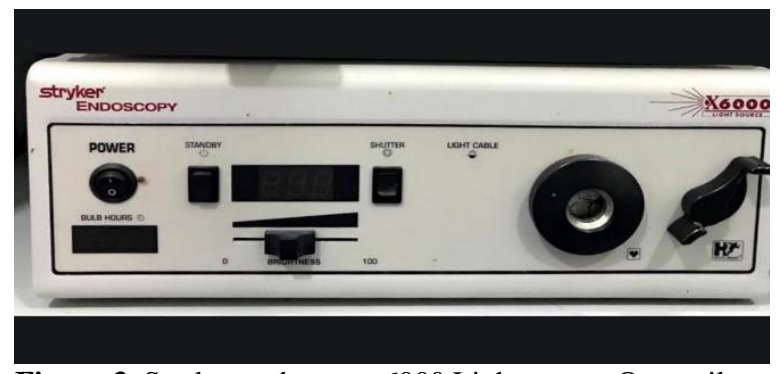

Figura 3. Stryker endoscopy 6000 Light source ${ }^{\circledR}$ para iluminação e visualização de estruturas no interior da cavidade.

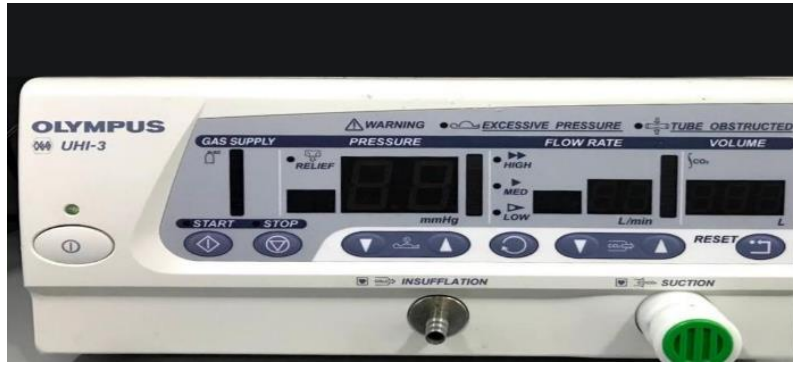

Figura 2. Insuflador Olympus UHI-3 ${ }^{\circledR}$ para expansão da cavidade abdominal.

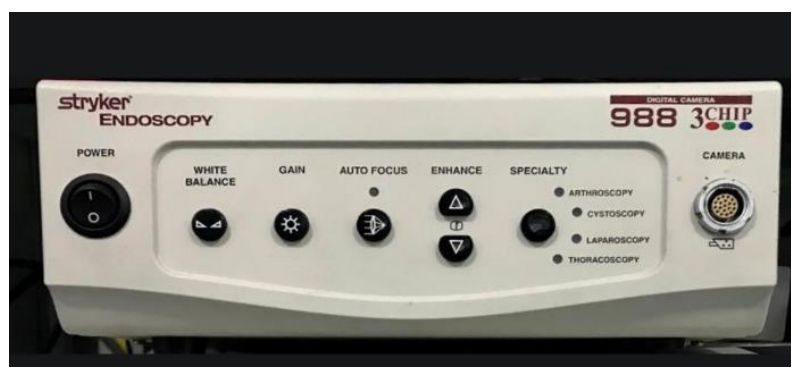

Figura 4. Stryker 988 Digital câmera ${ }^{\circledR}$ para visualização e gravação de procedimentos.

Após a inserção de ambos os portais (Figura 5), a paciente foi posicionada em decúbito lateral direito (Figura 6) para exposição do complexo ovariano esquerdo (Figura 7). O ovário foi elevado em direção ventral à parede abdominal lateral esquerda com o uso do ligasure Meryland - Valleylab ${ }^{\circledR}$ de $5 \mathrm{~mm}$ e $37 \mathrm{~cm}$ (Figura 8), por meio do segundo portal de $5 \mathrm{~mm}$, e através dele foi realizada manipulação, cauterização e corte.

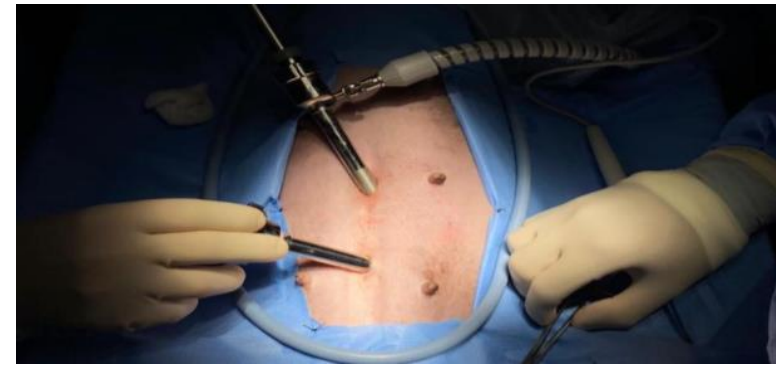

Figura 5. Inserção dos dois portais na cavidade abdominal.

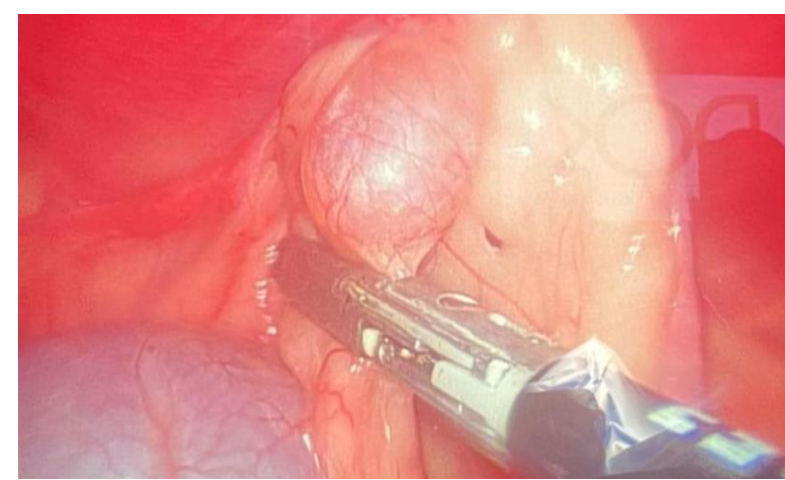

Figura 7. Exposição do complexo ovariano esquerdo com o ligasure.

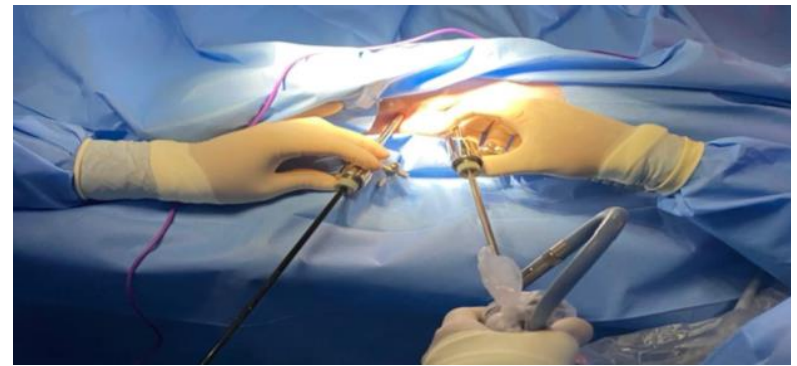

Figura 6. Paciente em decúbito lateral direito.

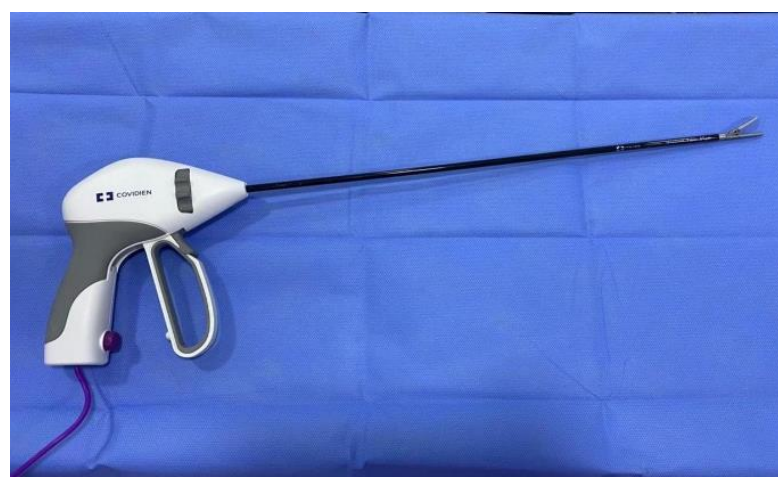

Figura 8. Ligasure Meryland - Valleylab ${ }^{\circledR}$.

Uma vez completa a secção e não evidenciado sangramento, o ovário foi liberado e retirado através do portal (Figura 9). Trocou-se o decúbito para lateral esquerdo, permitindo a visualização do complexo ovariano contralateral, sendo realizadas as mesmas etapas supracitadas. $\mathrm{O}$ mesmo permaneceu apreendido pela pinça e foi removido pelo portal do acesso pré-púbico. 
O tempo cirúrgico total foi de aproximadamente 40 minutos e o paciente recebeu alta $4 \mathrm{~h}$ após o procedimento. Durante a internação o paciente recebeu medicações via intravenosa (omeprazol, $1 \mathrm{mg} / \mathrm{kg}$, BID; ondansetrona $0,5 \mathrm{mg} / \mathrm{kg}$, TID; maxicam $0,1 \mathrm{mg} / \mathrm{kg}$ SID; dipirona $25 \mathrm{mg} / \mathrm{kg}$ TID). A cicatrização das feridas de acesso ocorreu 10 dias após o procedimento quando então foi realizada a remoção dos pontos.

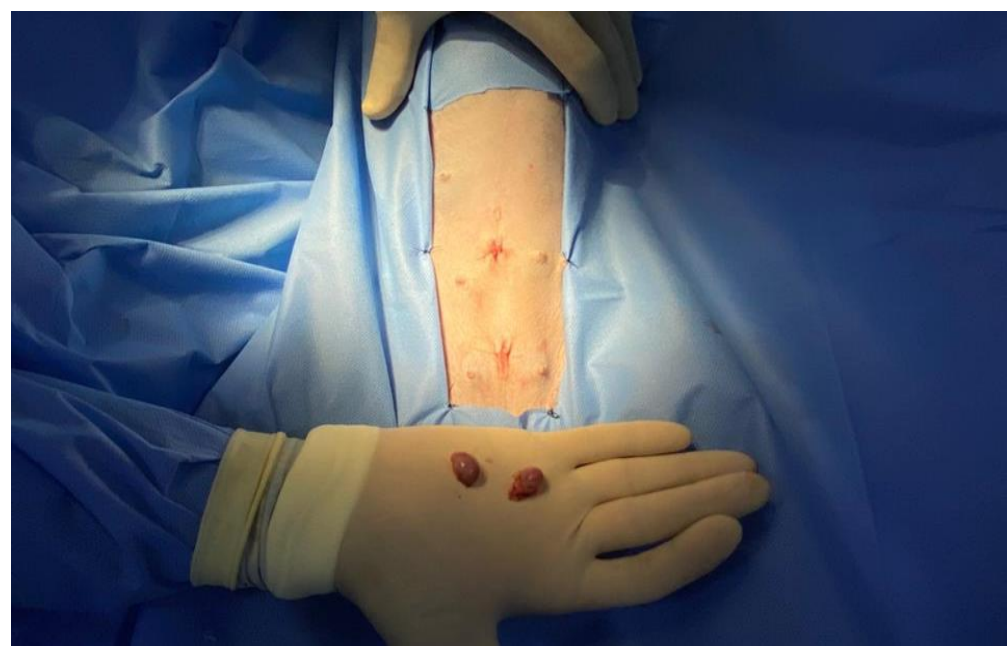

Figura 9. Ovários removidos na ovariectomia videolaparoscopica

\section{Discussão}

Segundo Hsueh et al. (2018), o tempo cirúrgico é de 13-22 minutos em uma equipe cirúrgica experiente, a presente equipe realizou o procedimento em 28 minutos. Como acontece com a maioria das novas técnicas cirúrgicas que usam tecnologia mais recente, espera-se que tempos operatórios mais longos e mais complicações relacionadas ao procedimento estejam associados aos estágios iniciais da curva de aprendizado. A equipe que realizou o procedimento participou de cursos fora do estado para aprender a técnica, visto que não é ministrado nos cursos de graduação veterinária uma matéria sobre vídeo cirurgia (Runge et al., 2014).

A comparação do custo e das variáveis clínicas associadas entre as técnicas laparoscópicas e cirúrgicas abertas é uma consideração importante em cirurgia humana e veterinária. $\mathrm{O}$ investimento é uma limitação segundo o autor Hsueh et al. (2018). No hospital em que foi realizada a cirurgia o equipamento foi adquirido através da equipe, é utilizado com rotina e tem boa aceitação dos clientes que, apesar do custo maior, entendem o benefício da técnica. Já em relação aos cirurgiões volantes, o preço do equipamento móvel com o equipamento fixo é um fator limitante para não desempenharem a vídeolaparoscopia em sua rotina.

Segundo Santos et al. (2020) a máxima visualização, menor trauma tecidual, diminuição da dor pósoperatória, menor tempo de recuperação pós-operatória, menor risco de infecção e redução do sangramento no transoperatório são vantagens discutidas e que também foram evidenciadas no presente estudo.

\section{Conclusão}

A ovariectomia por vídeolaparoscopica é uma opção viável para castração eletiva de fêmeas caninas saudáveis. É uma vantagem em relação aos métodos tradicionais em virtude de ser menos invasiva, o que permite melhor recuperação do paciente.

\section{Referências bibliográficas}

Carvalho, M. P. P., Koivisto, M. B., Perri, S. H. V., \& Sampaio, T. S. M. C. (2007). Estudo retrospectivo da esterilização em cães e gatos no município de Araçatuba, SP. Revista Ciência Em Extensão, 3(2), 81-94.

Ferraz, E. D., \& Lacombe, D. (2003). Estado atual da cirurgia híbrida colo-retal. Revista Brasileira de Video Cirúrgia, 1, 29-37. 
Hsueh, C., Giuffrida, M., Mayhew, P. D., Case, J. B., Singh, A., Monnet, E., Holt, D. E., Cray, M., Curcillo, C., \& Runge, J. J. (2018). Evaluation of pet owner preferences for operative sterilization techniques in female dogs within the veterinary community. Veterinary Surgery, 47(S1), 15-25.

Manassero, M., \& Viateau, V. (2018). Advances in laparoscopic spay techniques for dogs: the past, present and future. The Veterinary Record, 183(24), 742-744.

Nacul, M. P., Cavazzola, L. T., \& Melo, M. C. de. (2015). Situação atual do treinamento de médicos residentes em videocirurgia no Brasil: uma análise crítica. Arquivos Brasileiros de Cirurgia Digestiva, 28, 81-85. https://doi.org/10.1590/S0102-67202015000100020.

Runge, J. J., Boston, R. C., Ross, S. B., \& Brown, D. C. (2014). Evaluation of the learning curve for a board-certified veterinary surgeon performing laparoendoscopic single-site ovariectomy in dogs. Journal of the American Veterinary Medical Association, 245(7), 828-835. https://doi.org/10.2460/javma.245.7.828.

Santos, I. F. C., Apolonio, E. V. P., Gallina, M. F., Souza, P., Nishimaru, R., Almeida, K., Pereira, G., \& Sakata, S. H. (2020). Videocirurgia em cães e gatos - Revisão de literatura. Veterinária e Zootecnia, 27, 1-16. https://doi.org/10.35172/rvz.2020.v27.456.

Shariati, E., Bakhtiari, J., Khalaj, A., \& Niasari-Naslaji, A. (2014). Comparison between two portal laparoscopy and open surgery for ovariectomy in dogs. Veterinary Research Forum, 5(3), 219-223.

Venzin, C. (2014). Schonende Entfernung-Laproskopische Kastration bei der Hündin. Hundkatzepferd, $01 / 14,14-17$.

Histórico do artigo:

Recebido: 30 de setembro de 2021

Aprovado: 30 de outubro de 2021

Disponível online: 28 de fevereiro de 2022
Licenciamento: Este artigo é publicado na modalidade Acesso Aberto sob a licença Creative Commons Atribuição 4.0 (CC-BY 4.0), a qual permite uso irrestrito, distribuição, reprodução em qualquer meio, desde que o autor e a fonte sejam devidamente creditados. 